\title{
ON A DIRICHLET PROBLEM WITH $(p, q)$-LAPLACIAN AND PARAMETRIC CONCAVE-CONVEX NONLINEARITY
}

\author{
SALVATORE A. MARANO, GRETA MARINO, AND NIKOLAOS S. PAPAGEORGIOU
}

\begin{abstract}
A homogeneous Dirichlet problem with $(p, q)$-Laplace differential operator and reaction given by a parametric $p$-convex term plus a $q$-concave one is investigated. A bifurcation-type result, describing changes in the set of positive solutions as the parameter $\lambda>0$ varies, is proven. Since for every admissible $\lambda$ the problem has a smallest positive solution $\bar{u}_{\lambda}$, both monotonicity and continuity of the map $\lambda \mapsto \bar{u}_{\lambda}$ are studied.
\end{abstract}

\section{INTRODUCTION}

Let $\Omega$ be a bounded domain in $\mathbb{R}^{N}$ with a $C^{2}$-boundary $\partial \Omega$, let $1<\tau<q<p<+\infty$, and let $f: \Omega \times \mathbb{R} \rightarrow \mathbb{R}$ be a Carathéodory function. Consider the Dirichlet problem

$$
\begin{cases}-\Delta_{p} u-\Delta_{q} u=u^{\tau-1}+\lambda f(x, u) & \text { in } \Omega, \\ u>0 & \text { in } \Omega, \\ u=0 & \text { on } \partial \Omega,\end{cases}
$$

where $\lambda>0$ is a parameter while $\Delta_{r}, r>1$, denotes the $r$-Laplacian, namely

$$
\Delta_{r} u:=\operatorname{div}\left(|\nabla u|^{r-2} \nabla u\right) \quad \forall u \in W_{0}^{1, r}(\Omega) .
$$

The nonhomogeneous differential operator $A u:=\Delta_{p} u+\Delta_{q} u$ that drives $\left(\mathrm{P}_{\lambda}\right)$ is usually called $(p, q)$ Laplacian. It stems from a wide range of important applications, including models of elementary particles [8], biophysics [9], plasma physics [26], reaction-diffusion equations [7], elasticity theory [27], etc. That's why the relevant literature looks daily increasing and numerous meaningful works on this subject are by now available; see the survey paper [19] for a larger bibliography.

Since $\tau<q<p$, the function $\xi \mapsto \xi^{\tau-1}$ grows $(q-1)$-sublinearly at $+\infty$, whereas $\xi \mapsto f(x, \xi)$ is assumed to be $(p-1)$-superlinear near $+\infty$, although it need not satisfy the usual (in such cases) Ambrosetti-Rabinowitz condition. So, the reaction in $\left(\mathrm{P}_{\lambda}\right)$ exhibits the competing effects of concave and convex terms, with the latter multiplied by a positive parameter.

The aim of this paper is to investigate how the solution set of $\left(\mathrm{P}_{\lambda}\right)$ changes as $\lambda$ varies. In particular, we prove that there exists a critical parameter value $\lambda^{*}>0$ for which problem $\left(\mathrm{P}_{\lambda}\right)$ admits

- at least two solutions if $\lambda \in\left(0, \lambda^{*}\right)$,

- at least one solution when $\lambda=\lambda^{*}$, and

- no solution provided $\lambda>\lambda^{*}$.

Moreover, we detect a smallest positive solution $\bar{u}_{\lambda}$ for each $\lambda \in\left(0, \lambda^{*}\right]$ and show that the map $\lambda \mapsto \bar{u}_{\lambda}$ turns out left-continuous, besides increasing.

The first bifurcation result for semilinear Dirichlet problems driven by the Laplace operator was established, more than twenty years ago, in the seminal paper [2] and then extended to the $p$-Laplacian in $[11,16]$. These works treat the reaction

$$
\xi \mapsto \lambda \xi^{s-1}+\xi^{r-1}, \quad \xi \geq 0
$$

where $1<s<p<r<p^{*}, \lambda>0$, and $p^{*}$ denotes the critical Sobolev exponent. A wider class of nonlinearities has recently been investigated in [22], while [24] deals with Robin boundary conditions. It should be noted that, unlike our case, $\lambda$ always multiplies the concave term, which changes the

2010 Mathematics Subject Classification. 35J20, 35J60.

Key words and phrases. $(p, q)$-Laplacian, concave-convex nonlinearity, positive solution, bifurcation-type theorem. 
analysis of the problem. Finally, $[4,14,23]$ contain analogous bifurcation theorems for problems of a different kind, whereas $[20,21]$ study $(p, q)$-Laplace equations having merely concave right-hand side.

Our approach is based on the critical point theory, combined with appropriate truncation and comparison techniques.

\section{MAThematicAl BACKGROUND AND HYPOTHESES}

Let $(X,\|\cdot\|)$ be a real Banach space. Given a set $V \subseteq X$, write $\bar{V}$ for the closure of $V, \partial V$ for the boundary of $V$, and $\operatorname{int}_{X}(V)$ or simply $\operatorname{int}(V)$, when no confusion can arise, for the interior of $V$. If $x \in X$ and $\delta>0$ then

$$
B_{\delta}(x):=\{z \in X:\|z-x\|<\delta\}, \quad B_{\delta}:=B_{\delta}(0) .
$$

The symbol $\left(X^{*},\|\cdot\|_{X^{*}}\right)$ denotes the dual space of $X,\langle\cdot, \cdot\rangle$ indicates the duality pairing between $X$ and $X^{*}$, while $x_{n} \rightarrow x$ (respectively, $x_{n} \rightarrow x$ ) in $X$ means the sequence $\left\{x_{n}\right\}$ converges strongly (respectively, weakly) in $X$ '. We say that $A: X \rightarrow X^{*}$ is of type $(\mathrm{S})_{+}$provided

$$
x_{n} \rightarrow x \text { in } X, \quad \limsup _{n \rightarrow+\infty}\left\langle A\left(x_{n}\right), x_{n}-x\right\rangle \leq 0 \quad \Longrightarrow \quad x_{n} \rightarrow x .
$$

The function $\Phi: X \rightarrow \mathbb{R}$ is called coercive if $\lim _{\|x\| \rightarrow+\infty} \Phi(x)=+\infty$ and weakly sequentially lower semicontinuous when

$$
x_{n} \rightarrow x \text { in } X \quad \Longrightarrow \quad \Phi(x) \leq \liminf _{n \rightarrow \infty} \Phi\left(x_{n}\right) .
$$

Suppose $\Phi \in C^{1}(X)$. We denote by $K(\Phi)$ the critical set of $\Phi$, i.e.,

$$
K(\Phi):=\left\{x \in X: \Phi^{\prime}(x)=0\right\} .
$$

The classical Cerami compactness condition for $\Phi$ reads as follows:

(C) Every $\left\{x_{n}\right\} \subseteq X$ such that $\left\{\Phi\left(x_{n}\right)\right\}$ is bounded and $\left(1+\left\|x_{n}\right\|\right) \Phi^{\prime}\left(x_{n}\right) \rightarrow 0$ in $X^{*}$ has a convergent subsequence.

From now on, $\Omega$ indicates a fixed bounded domain in $\mathbb{R}^{N}$ with a $C^{2}$-boundary $\partial \Omega$. Let $u, v: \Omega \rightarrow \mathbb{R}$ be measurable and let $t \in \mathbb{R}$. The symbol $u \leq v$ means $u(x) \leq v(x)$ for almost every $x \in \Omega$, $t^{ \pm}:=\max \{ \pm t, 0\}, u^{ \pm}(\cdot):=u(\cdot)^{ \pm}$. If $u, v$ belong to a function space, say $Y$, then we set

$$
[u, v]:=\{w \in Y: u \leq w \leq v\}, \quad[u):=\{w \in Y: u \leq w\} .
$$

The conjugate exponent $r^{\prime}$ of a number $r \geq 1$ is defined by $r^{\prime}:=r /(r-1)$, while $r^{*}$ indicates its Sobolev conjugate, namely

As usual,

$$
r^{*}:= \begin{cases}\frac{N r}{N-r} & \text { when } r<N \\ +\infty & \text { otherwise }\end{cases}
$$

$$
\|u\|_{r}:=\left(\int_{\Omega}|u|^{r} d x\right)^{1 / r} \forall u \in L^{r}(\Omega), \quad\|u\|_{1, r}:=\left(\int_{\Omega}|\nabla u|^{r} d x\right)^{1 / r} \forall u \in W_{0}^{1, r}(\Omega),
$$

and $W^{-1, r^{\prime}}(\Omega)$ denotes the dual space of $W_{0}^{1, r}(\Omega)$. We will also employ the linear space $C_{0}^{1}(\bar{\Omega}):=$ $\left\{u \in C^{1}(\bar{\Omega}): u\lfloor\partial \Omega=0\}\right.$, which is complete with respect to the standard $C^{1}(\bar{\Omega})$-norm. Its positive cone

$$
C_{+}:=\left\{u \in C_{0}^{1}(\bar{\Omega}): u(x) \geq 0 \text { in } \bar{\Omega}\right\}
$$

has a nonempty interior given by

$$
\operatorname{int}\left(C_{+}\right)=\left\{u \in C_{+}: u(x)>0 \forall x \in \Omega, \frac{\partial u}{\partial n}(x)<0 \forall x \in \partial \Omega\right\} .
$$

Here $n(x)$ denotes the outward unit normal to $\partial \Omega$ at $x$.

Let $A_{r}: W_{0}^{1, r}(\Omega) \rightarrow W^{-1, r^{\prime}}(\Omega)$ be the nonlinear operator stemming from the negative $r$-Laplacian, i.e.,

$$
\left\langle A_{r}(u), v\right\rangle:=\int_{\Omega}|\nabla u|^{r-2} \nabla u \cdot \nabla v d x, \quad u, v \in W_{0}^{1, r}(\Omega) .
$$


We know [12, Section 6.2] that $A_{r}$ is bounded, continuous, strictly monotone, and of type $(\mathrm{S})_{+}$. The Liusternik-Schnirelmann theory gives an increasing sequence $\left\{\lambda_{n, r}\right\}$ of eigenvalues for $A_{r}$. The following assertions can be found in [12, Section 6.2].

$\left(\mathrm{p}_{1}\right) \lambda_{1, r}$ is positive, isolated, and simple.

$\left(\mathrm{p}_{2}\right)\|u\|_{r}^{r} \leq \frac{1}{\lambda_{1, r}}\|u\|_{1, r}^{r}$ for all $u \in W_{0}^{1, r}(\Omega)$.

$\left(\mathrm{p}_{3}\right) \lambda_{1, r}$ admits an eigenfunction $\phi_{1, r} \in \operatorname{int}\left(C_{+}\right)$such that $\left\|\phi_{1, r}\right\|_{r}=1$.

Proposition 13 of [6] then ensures that

$\left(\mathrm{p}_{4}\right)$ If $r \neq \hat{r}$ then $\phi_{1, r}$ and $\phi_{1, \hat{r}}$ are linearly independent.

Let $g: \Omega \times \mathbb{R} \rightarrow \mathbb{R}$ be a Carathéodory function satisfying the growth condition

$$
|g(x, t)| \leq a(x)\left(1+|t|^{s-1}\right) \text { in } \Omega \times \mathbb{R},
$$

where $a \in L^{\infty}(\mathbb{R}), 1<s \leq p^{*}$. Set $G(x, \xi):=\int_{0}^{\xi} g(x, t) d t$ and consider the $C^{1}$-functional $\varphi$ : $W_{0}^{1, p}(\Omega) \rightarrow \mathbb{R}$ defined by

$$
\varphi(u):=\frac{1}{p}\|\nabla u\|_{p}^{p}+\frac{1}{q}\|\nabla u\|_{q}^{q}-\int_{\Omega} G(x, u(x)) d x, \quad u \in W_{0}^{1, p}(\Omega) .
$$

Proposition 2.1 ( [13], Proposition 2.6). If $u_{0} \in W_{0}^{1, p}(\Omega)$ is a local $C_{0}^{1}(\bar{\Omega})$-minimizer of $\varphi$ then $u_{0} \in C^{1, \alpha}(\bar{\Omega})$ for some $\alpha \in(0,1)$ and $u_{0}$ turns out to be a local $W_{0}^{1, p}(\Omega)$-minimizer of $\varphi$.

Combining this result with the strong comparison principle below, essentially due to Arcoya-Ruiz [3], shows that certain constrained minimizers actually are 'global' critical points. Recall that, given $h_{1}, h_{2} \in L^{\infty}(\Omega)$,

$$
h_{1} \prec h_{2} \Longleftrightarrow \underset{K}{\operatorname{ess} \inf }\left(h_{2}-h_{1}\right)>0 \text { for any nonempty compact set } K \subseteq \Omega \text {. }
$$

Proposition 2.2. Let $a \in \mathbb{R}_{+}, h_{1}, h_{2} \in L^{\infty}(\Omega), u_{1} \in C_{0}^{1}(\bar{\Omega}), u_{2} \in \operatorname{int}\left(C_{+}\right)$. Suppose $h_{1} \prec h_{2}$ as well as

$$
-\Delta_{p} u_{i}-\Delta_{q} u_{i}+a\left|u_{i}\right|^{p-2} u_{i}=h_{i} \text { in } \Omega, \quad i=1,2 .
$$

Then, $u_{2}-u_{1} \in \operatorname{int}\left(C_{+}\right)$.

Throughout the paper, 'for every $x \in \Omega$ ' will take the place of 'for almost every $x \in \Omega$ ', $c_{0}, c_{1}, \ldots$ indicate suitable positive constants, $f: \Omega \times \mathbb{R} \rightarrow \mathbb{R}$ is a Carathéodory function such that $f(\cdot, t)=0$ provided $t \leq 0$, while $F(x, \xi):=\int_{0}^{\xi} f(x, t) d t$.

The following hypotheses will be posited.

$\left(\mathrm{h}_{1}\right)$ There exist $\theta \in[\tau, q]$ and $r \in\left(p, p^{*}\right)$ such that

$$
c_{1} t^{p-1}+c_{2} t^{q-1} \leq f(x, t) \leq c_{0}\left(t^{\theta-1}+t^{r-1}\right) \quad \forall(x, t) \in \Omega \times \mathbb{R}_{+},
$$

where $c_{2}>\lambda_{1, q}$

$\left(\mathrm{h}_{2}\right) \lim _{\xi \rightarrow+\infty} \frac{F(x, \xi)}{\xi^{p}}=+\infty$ uniformly with respect to $x \in \Omega$.

$\left(\mathrm{h}_{3}\right) \liminf _{\xi \rightarrow+\infty} \frac{f(x, \xi) \xi-p F(x, \xi)}{\xi^{\beta}} \geq c_{3}$ uniformly in $x \in \Omega$. Here, $\beta>\tau$ and

$$
(r-p) \max \left\{N p^{-1}, 1\right\}<\beta<p^{*} .
$$

$\left(\mathrm{h}_{4}\right)$ To every $\rho>0$ there corresponds $\mu_{\rho}>0$ such that $t \mapsto f(x, t)+\mu_{\rho} t^{p-1}$ is nondecreasing in $[0, \rho]$ for any $x \in \Omega$.

By $\left(\mathrm{h}_{2}\right)-\left(\mathrm{h}_{3}\right)$ the perturbation $f(x, \cdot)$ is $(p-1)$-superlinear at $+\infty$. In the literature, one usually treats this case via the well-known Ambrosetti-Rabinowitz condition, namely:

(AR) With appropriate $M>0, \sigma>p$ one has both ess $\inf F(\cdot, M)>0$ and

$$
0<\sigma F(x, \xi) \leq f(x, \xi) \xi, \quad(x, \xi) \in \Omega \times[M,+\infty) .
$$


It easily entails $c_{3} \xi^{\sigma} \leq F(x, \xi)$ in $\Omega \times[M,+\infty)$, which forces $\left(\mathrm{h}_{2}\right)$. However, nonlinearities having a growth rate 'slower' than $t^{\sigma-1}$ at $+\infty$ are excluded from (2.1). Thus, assumption $\left(\mathrm{h}_{3}\right)$ incorporates in our framework more situations.

Example 2.3. Let $c_{2}>\lambda_{1, q}$. The functions $f_{1}, f_{2}: \mathbb{R}_{+} \rightarrow \mathbb{R}$ defined by

$$
f_{1}(t):=\left\{\begin{array}{ll}
t^{p-1}+c_{2} t^{\tau-1} & \text { if } 0 \leq t \leq 1, \\
t^{r-1}+c_{2} t^{q-1} & \text { otherwise, }
\end{array} \quad f_{2}(t):=t^{p-1} \log (1+t)+c_{2} t^{q-1}, \quad t \in \mathbb{R}_{+},\right.
$$

satisfy $\left(\mathrm{h}_{1}\right)-\left(\mathrm{h}_{4}\right)$. Nevertheless, $f_{1}$ alone complies with condition $(A R)$.

\section{A bifurcation-type theorem}

Write $S_{\lambda}$ for the set of positive solutions to $\left(\mathrm{P}_{\lambda}\right)$. Lieberman's nonlinear regularity theory [18, $\mathrm{p}$. $320]$ and Pucci-Serrin's maximum principle [25, pp. 111,120] yield

$$
S_{\lambda} \subseteq \operatorname{int}\left(C_{+}\right) .
$$

Put $\mathcal{L}:=\left\{\lambda>0: S_{\lambda} \neq \emptyset\right\}$. Our first goal is to establish some basic properties of $\mathcal{L}$. From now on, $X:=W_{0}^{1, p}(\Omega)$ and $\|\cdot\|:=\|\cdot\|_{1, p}$.

Proposition 3.1. Under $\left(\mathrm{h}_{1}\right)$ one has $\mathcal{L} \neq \emptyset$.

Proof. Given $\lambda>0$, consider the $C^{1}$-functional $\Psi_{\lambda}: W_{0}^{1, p}(\Omega) \rightarrow \mathbb{R}$ defined by

$$
\Psi_{\lambda}(u):=\frac{1}{p}\|\nabla u\|_{p}^{p}+\frac{1}{q}\|\nabla u\|_{q}^{q}-\int_{\Omega} d x \int_{0}^{u(x)} g_{\lambda}(t) d t \quad \forall u \in W_{0}^{1, p}(\Omega),
$$

where

$$
g_{\lambda}(t):=\left(t^{+}\right)^{\tau-1}+\lambda c_{0}\left[\left(t^{+}\right)^{\theta-1}+\left(t^{+}\right)^{r-1}\right], \quad t \in \mathbb{R} .
$$

Evidently, $g_{\lambda}$ fulfills (2.1) once $\sigma \in(p, r)$ and $M>0$ is big enough. So, condition (C) holds true for $\Psi_{\lambda}$. Moreover,

$$
u \in \operatorname{int}\left(C_{+}\right) \Longrightarrow \lim _{t \rightarrow+\infty} \Psi_{\lambda}(t u)=-\infty
$$

because $r>p$. Observe next that if $s \in\left[1, p^{*}\right]$ then

$$
\|u\|_{s} \leq c\|u\|_{p^{*}} \leq C\|u\| \quad \forall u \in X,
$$

with $C:=C(s, \Omega)$. This easily leads to

$$
\begin{aligned}
\Psi_{\lambda}(u) & \geq \frac{1}{p}\|u\|^{p}-c_{4}\|u\|^{\tau}-\lambda c_{5}\left[\|u\|^{\theta}+\|u\|^{r}\right] \\
& =\left[\frac{1}{p}-c_{4}\|u\|^{\tau-p}-\lambda c_{5}\left(\|u\|^{\theta-p}+\|u\|^{r-p}\right)\right]\|u\|^{p}, \quad u \in X .
\end{aligned}
$$

Let us set, for any $t>0$,

$$
\gamma_{\lambda}(t):=c_{4} t^{\tau-p}+\lambda c_{5}\left(t^{\theta-p}+t^{r-p}\right), \quad \hat{\gamma}_{\lambda}(t):=\left(c_{4}+\lambda c_{5}\right) t^{\tau-p}+2 \lambda c_{5} t^{r-p} .
$$

From $\tau \leq \theta<p<r$ it follows $\lambda c_{5} t^{\theta-p} \leq \lambda c_{5}\left(t^{\tau-p}+t^{r-p}\right)$, which implies

$$
0<\gamma_{\lambda}(t) \leq \hat{\gamma}_{\lambda}(t) \quad \text { in } \quad(0,+\infty)
$$

Since $\lim _{t \rightarrow 0^{+}} \hat{\gamma}_{\lambda}(t)=\lim _{t \rightarrow+\infty} \hat{\gamma}_{\lambda}(t)=+\infty$, there exists $t_{0}>0$ satisfying $\hat{\gamma}_{\lambda}^{\prime}\left(t_{0}\right)=0$. One has

$$
t_{0}:=t_{0}(\lambda):=\left[\frac{\left(c_{4}+\lambda c_{5}\right)(p-\tau)}{2 \lambda c_{5}(r-p)}\right]^{\frac{1}{r-\tau}}
$$

and, via simple calculations, $\lim _{\lambda \rightarrow 0^{+}} \hat{\gamma}_{\lambda}\left(t_{0}\right)=0$. On account of (3.1)-(3.2) we can thus find $\lambda_{0}>0$ such that

$$
\Psi_{\lambda}(u) \geq m_{\lambda}>0=\Psi_{\lambda}(0) \quad \text { for all } u \in \partial B\left(0, t_{0}\right), \lambda \in\left(0, \lambda_{0}\right) .
$$


Pick $\lambda \in\left(0, \lambda_{0}\right)$. The mountain pass theorem entails $\Psi_{\lambda}^{\prime}\left(\bar{u}_{\lambda}\right)=0$ and $\Psi_{\lambda}\left(\bar{u}_{\lambda}\right) \geq m_{\lambda}$ with appropriate $\bar{u}_{\lambda} \in X$. Hence,

$$
\left\langle A_{p}\left(\bar{u}_{\lambda}\right)+A_{q}\left(\bar{u}_{\lambda}\right), v\right\rangle=\int_{\Omega}\left[\left(\bar{u}_{\lambda}^{+}\right)^{\tau-1}+\lambda c_{0}\left(\left(\bar{u}_{\lambda}^{+}\right)^{\theta-1}+\left(\bar{u}_{\lambda}^{+}\right)^{r-1}\right)\right] v d x, \quad v \in X,
$$

and $\bar{u}_{\lambda} \neq 0$. Choosing $v:=-\bar{u}_{\lambda}^{-}$in (3.3) yields $\left\|\nabla \bar{u}_{\lambda}^{-}\right\|_{p}^{p}+\left\|\nabla \bar{u}_{\lambda}^{-}\right\|_{q}^{q}=0$, namely $\bar{u}_{\lambda}^{-}=0$. This forces $\bar{u}_{\lambda} \geq 0$ while, by (3.3) again,

$$
-\Delta_{p} \bar{u}_{\lambda}-\Delta_{q} \bar{u}_{\lambda}=\bar{u}_{\lambda}^{\tau-1}+\lambda c_{0}\left(\bar{u}_{\lambda}^{\theta-1}+\bar{u}_{\lambda}^{r-1}\right) \text { in } \Omega .
$$

Lieberman's nonlinear regularity theory and Pucci-Serrin's maximum principle finally lead to $\bar{u}_{\lambda} \in$ $\operatorname{int}\left(C_{+}\right)$. Now define, provided $(x, \xi) \in \Omega \times \mathbb{R}$,

$$
\bar{f}_{\lambda}(x, \xi):= \begin{cases}\left(\xi^{+}\right)^{\tau-1}+\lambda f\left(x, \xi^{+}\right) & \text {if } \xi \leq \bar{u}_{\lambda}(x), \quad \bar{F}_{\lambda}(x, \xi):=\int_{0}^{\xi} \bar{f}_{\lambda}(x, t) d t . \\ \bar{u}_{\lambda}(x)^{\tau-1}+\lambda f\left(x, \bar{u}_{\lambda}(x)\right) & \text { otherwise, }\end{cases}
$$

An easy verification ensures that the associated $C^{1}$-functional

$$
\bar{\Phi}_{\lambda}(u):=\frac{1}{p}\|\nabla u\|_{p}^{p}+\frac{1}{q}\|\nabla u\|_{q}^{q}-\int_{\Omega} \bar{F}_{\lambda}(x, u(x)) d x, \quad u \in X,
$$

is coercive and weakly sequentially lower semicontinuous. So, it attains its infimum at some point $u_{\lambda} \in X$. Assumption $\left(\mathrm{h}_{1}\right)$ produces

$$
\bar{\Phi}_{\lambda}\left(u_{\lambda}\right)<0=\bar{\Phi}_{\lambda}(0),
$$

i.e., $u_{\lambda} \neq 0$, because $\tau<q<p$. As before, from

$$
\left\langle A_{p}\left(u_{\lambda}\right)+A_{q}\left(u_{\lambda}\right), v\right\rangle=\int_{\Omega} \bar{f}_{\lambda}\left(x, u_{\lambda}(x)\right) v(x) d x \quad \forall v \in X
$$

we infer $u_{\lambda} \geq 0$. Test (3.4) with $v:=\left(u_{\lambda}-\bar{u}_{\lambda}\right)^{+}$, exploit $\left(\mathrm{h}_{1}\right)$ again, and recall (3.3) to arrive at

$$
\begin{aligned}
\left\langle A_{p}\left(u_{\lambda}\right)+A_{q}\left(u_{\lambda}\right),\left(u_{\lambda}-\bar{u}_{\lambda}\right)^{+}\right\rangle & =\int_{\Omega}\left[\bar{u}_{\lambda}^{\tau-1}+\lambda f\left(\cdot, \bar{u}_{\lambda}\right)\right]\left(u_{\lambda}-\bar{u}_{\lambda}\right)^{+} d x \\
& \leq \int_{\Omega}\left[\bar{u}_{\lambda}^{\tau-1}+\lambda c_{0}\left(\bar{u}_{\lambda}^{\theta-1}+\bar{u}_{\lambda}^{r-1}\right)\right]\left(u_{\lambda}-\bar{u}_{\lambda}\right)^{+} d x \\
& =\left\langle A_{p}\left(\bar{u}_{\lambda}\right)+A_{q}\left(\bar{u}_{\lambda}\right),\left(u_{\lambda}-\bar{u}_{\lambda}\right)^{+}\right\rangle,
\end{aligned}
$$

which entails $u_{\lambda} \leq \bar{u}_{\lambda}$ by monotonicity. Summing up, $u_{\lambda} \in\left[0, \bar{u}_{\lambda}\right] \backslash\{0\}$. On account of (3.4), one thus has $u_{\lambda} \in S_{\lambda}$ for any $\lambda \in\left(0, \lambda_{0}\right)$. This completes the proof.

Our next result ensures that $\mathcal{L}$ is an interval.

Proposition 3.2. Let $\left(\mathrm{h}_{1}\right)$ be satisfied. If $\hat{\lambda} \in \mathcal{L}$ then $(0, \hat{\lambda}) \subseteq \mathcal{L}$.

Proof. Pick $\hat{u} \in S_{\hat{\lambda}}, \lambda \in(0, \hat{\lambda})$, and define, provided $(x, \xi) \in \Omega \times \mathbb{R}$,

$$
\hat{f}_{\lambda}(x, \xi):=\left\{\begin{array}{ll}
\left(\xi^{+}\right)^{\tau-1}+\lambda f\left(x, \xi^{+}\right) & \text {if } \xi \leq \hat{u}(x), \\
\hat{u}(x)^{\tau-1}+\lambda f(x, \hat{u}(x)) & \text { otherwise, }
\end{array} \quad \hat{F}_{\lambda}(x, \xi):=\int_{0}^{\xi} \hat{f}_{\lambda}(x, t) d t .\right.
$$

The associated energy functional

$$
\hat{\Phi}_{\lambda}(u):=\frac{1}{p}\|\nabla u\|_{p}^{p}+\frac{1}{q}\|\nabla u\|_{q}^{q}-\int_{\Omega} \hat{F}_{\lambda}(x, u(x)) d x, \quad u \in X
$$

turns out coercive, weakly sequentially lower semicontinuous, besides $C^{1}$. Now, arguing exactly as above yields the conclusion.

A careful reading of this proof allows one to state the next 'monotonicity' property.

Corollary 3.3. Under hypothesis $\left(\mathrm{h}_{1}\right)$, for every $\hat{\lambda} \in \mathcal{L}, u_{\hat{\lambda}} \in S_{\hat{\lambda}}$, and $\lambda \in(0, \hat{\lambda})$ there exists $u_{\lambda} \in S_{\lambda}$ such that $u_{\lambda} \leq u_{\hat{\lambda}}$.

Actually, we can prove a more precise assertion. 
Proposition 3.4. Suppose $\left(\mathrm{h}_{1}\right)$ and $\left(\mathrm{h}_{4}\right)$ hold. Then to each $\hat{\lambda} \in \mathcal{L}, u_{\hat{\lambda}} \in S_{\hat{\lambda}}, \lambda \in(0, \hat{\lambda})$ there corresponds $u_{\lambda} \in S_{\lambda}$ fulfilling $u_{\hat{\lambda}}-u_{\lambda} \in \operatorname{int}\left(C_{+}\right)$.

Proof. Write $\rho:=\left\|u_{\hat{\lambda}}\right\|_{\infty}$. If $\mu_{\rho}$ is given by $\left(\mathrm{h}_{4}\right)$ while $u_{\lambda}$ comes from Corollary 3.3 then

$$
\begin{aligned}
-\Delta_{p} u_{\hat{\lambda}} & -\Delta_{q} u_{\hat{\lambda}}+\lambda \mu_{\rho} u_{\hat{\lambda}}^{p-1}=u_{\hat{\lambda}}^{\tau-1}+\hat{\lambda} f\left(x, u_{\hat{\lambda}}\right)+\lambda \mu_{\rho} u_{\hat{\lambda}}^{p-1} \\
& =u_{\hat{\lambda}}^{\tau-1}+\lambda f\left(x, u_{\hat{\lambda}}\right)+\lambda \mu_{\rho} u_{\hat{\lambda}}^{p-1}+(\hat{\lambda}-\lambda) f\left(x, u_{\hat{\lambda}}\right) \\
& \geq u_{\lambda}^{\tau-1}+\lambda f\left(x, u_{\lambda}\right)+\lambda \mu_{\rho} u_{\lambda}^{p-1}=-\Delta_{p} u_{\lambda}-\Delta_{q} u_{\lambda}+\lambda \mu_{\rho} u_{\lambda}^{p-1}
\end{aligned}
$$

because $u_{\lambda} \leq u_{\hat{\lambda}}$ and $f(x, t) \geq 0$ once $t \geq 0$. The function $h(x):=(\hat{\lambda}-\lambda) f\left(x, u_{\hat{\lambda}}(x)\right)$ lies in $L^{\infty}(\Omega)$. Indeed, on account of $\left(\mathrm{h}_{1}\right)$, we have

$$
0 \leq h(x) \leq c_{0}(\hat{\lambda}-\lambda)\left[\|u\|_{\infty}^{\theta-1}+\|u\|_{\infty}^{r-1}\right] \quad \forall x \in \Omega .
$$

Pick any compact set $K \subseteq \Omega$. Recalling that $u_{\hat{\lambda}} \in \operatorname{int}\left(C_{+}\right)$and using $\left(\mathrm{h}_{1}\right)$ again gives

$$
h(x) \geq(\hat{\lambda}-\lambda)\left[c_{1} u_{\hat{\lambda}}(x)^{p-1}+c_{2} u_{\hat{\lambda}}(x)^{q-1}\right] \geq\left(c_{1} \inf _{K} u_{\hat{\lambda}}^{p-1}+c_{2} \inf _{K} u_{\hat{\lambda}}^{q-1}\right)>0, \quad x \in \Omega,
$$

whence $0 \prec h$. Now, (3.5) combined with Proposition 2.2 entail $u_{\hat{\lambda}}-u_{\lambda} \in \operatorname{int}\left(C_{+}\right)$.

The interval $\mathcal{L}$ turns out to be bounded.

Proposition 3.5. Let $\left(\mathrm{h}_{1}\right)$ and $\left(\mathrm{h}_{4}\right)$ be satisfied. If $\lambda^{*}:=\sup \mathcal{L}$ then $\lambda^{*}<\infty$.

Proof. Fix $\lambda \in \mathcal{L}, u_{\lambda} \in S_{\lambda}$. Note that we can suppose $\lambda>1$, otherwise $\mathcal{L}$ would be bounded, which of course entails $\lambda^{*}<\infty$. Define

$$
g_{\lambda}(x, \xi):=\left\{\begin{array}{ll}
\lambda\left[c_{1}\left(\xi^{+}\right)^{p-1}+c_{2}\left(\xi^{+}\right)^{q-1}\right] & \text { if } \xi \leq u_{\lambda}(x), \\
\lambda\left[c_{1} u_{\lambda}(x)^{p-1}+c_{2} u_{\lambda}(x)^{q-1}\right] & \text { otherwise, }
\end{array} G_{\lambda}(x, \xi):=\int_{0}^{\xi} g_{\lambda}(x, t) d t\right.
$$

for every $(x, \xi) \in \Omega \times \mathbb{R}$, as well as

$$
\Psi_{\lambda}(u):=\frac{1}{p}\|\nabla u\|_{p}^{p}+\frac{1}{q}\|\nabla u\|_{q}^{q}-\int_{\Omega} G_{\lambda}(x, u(x)) d x, \quad u \in X .
$$

The same arguments employed before yield here a global minimum point, say $\bar{u}_{\lambda}$, to $\Psi_{\lambda}$. So, in particular,

$$
\left\langle A_{p}\left(\bar{u}_{\lambda}\right)+A_{q}\left(\bar{u}_{\lambda}\right), v\right\rangle=\int_{\Omega} g_{\lambda}\left(x, \bar{u}_{\lambda}(x)\right) v(x) d x \quad \forall v \in X .
$$

Choosing $v:=-\bar{u}_{\lambda}^{-}$first and then $v:=\left(\bar{u}_{\lambda}-u_{\lambda}\right)^{+}$we obtain $\bar{u}_{\lambda} \in\left[0, u_{\lambda}\right]$; cf. the proof of Proposition 3.1. Since, by $\left(\mathrm{p}_{3}\right)$ in Section $2, u_{\lambda}, \phi_{1, q} \in \operatorname{int}\left(C_{+}\right)$, through [22, Proposition 1] one has $t \phi_{1, q} \leq u_{\lambda}$, with $t>0$ small enough. Thus, on account of $\left(\mathrm{p}_{3}\right)$ again,

$$
\begin{aligned}
\Psi_{\lambda}\left(t \phi_{1, q}\right) & =\frac{1}{p}\left\|\nabla\left(t \phi_{1, q}\right)\right\|_{p}^{p}+\frac{1}{q}\left\|\nabla\left(t \phi_{1, q}\right)\right\|_{q}^{q}-\int_{\Omega} G_{\lambda}\left(x, t \phi_{1, q}(x)\right) d x \\
& =\frac{t^{p}}{p}\left\|\nabla \phi_{1, q}\right\|_{p}^{p}+\frac{t^{q}}{q}\left\|\nabla \phi_{1, q}\right\|_{q}^{q}-\int_{\Omega} \lambda\left(c_{1} \frac{t^{p}}{p} \phi_{1, q}^{p}+c_{2} \frac{t^{q}}{q} \phi_{1, q}^{q}\right) d x \\
& =\frac{t^{p}}{p}\left\|\nabla \phi_{1, q}\right\|_{p}^{p}+\frac{t^{q}}{q} \lambda_{1, q}-\lambda c_{1} \frac{t^{p}}{p}\left\|\phi_{1, q}\right\|_{p}^{p}-\lambda c_{2} \frac{t^{q}}{q} \\
& \leq \frac{t^{p}}{p}\left\|\nabla \phi_{1, q}\right\|_{p}^{p}+\frac{t^{q}}{q}\left(\lambda_{1, q}-\lambda c_{2}\right) \\
& <\frac{t^{p}}{p}\left\|\nabla \phi_{1, q}\right\|_{p}^{p}+\frac{t^{q}}{q} \lambda_{1, q}(1-\lambda)=c_{6} t^{p}-c_{7} t^{q} .
\end{aligned}
$$

Now, recall that $q<p$ and decrease $t$ when necessary to achieve

$$
\Psi_{\lambda}\left(\bar{u}_{\lambda}\right)=\min _{X} \Psi_{\lambda} \leq \Psi_{\lambda}\left(t \phi_{1, q}\right)<0=\Psi_{\lambda}(0),
$$


i.e., $\bar{u}_{\lambda} \neq 0$. Summing up, $\bar{u}_{\lambda} \in\left[0, u_{\lambda}\right] \backslash\{0\}$, whence, by (3.6), it turns out a positive solution of the equation

$$
-\Delta_{p} u-\Delta_{q} u=\lambda c_{1}|u|^{p-2} u+\lambda c_{2}|u|^{q-2} u \text { in } \Omega .
$$

Due to [5, Theorem 2.4], this prevents $\lambda$ from being arbitrary large, as desired.

Le us finally prove that $\mathcal{L}=\left(0, \lambda^{*}\right]$. From now on, $\Phi_{\lambda}: X \rightarrow \mathbb{R}$ will denote the $C^{1}$-energy functional associated with problem $\left(\mathrm{P}_{\lambda}\right)$. Evidently,

$$
\Phi_{\lambda}(u)=\frac{1}{p}\|\nabla u\|_{p}^{p}+\frac{1}{q}\|\nabla u\|_{q}^{q}-\frac{1}{\tau}\left\|u^{+}\right\|_{\tau}^{\tau}-\lambda \int_{\Omega} F\left(x, u^{+}(x)\right) d x \quad \forall u \in X .
$$

Proposition 3.6. Under $\left(\mathrm{h}_{1}\right),\left(\mathrm{h}_{3}\right)$, and $\left(\mathrm{h}_{4}\right)$ one has $\lambda^{*} \in \mathcal{L}$.

Proof. Pick any $\left\{\lambda_{n}\right\} \subseteq\left(0, \lambda^{*}\right)$ fulfilling $\lambda_{n} \uparrow \lambda^{*}$. Via Corollary 3.3, construct a sequence $\left\{u_{n}\right\} \subseteq X$ such that $u_{n} \in S_{\lambda_{n}}, u_{n} \leq u_{n+1}$. Then

$$
\left\langle A_{p}\left(u_{n}\right)+A_{q}\left(u_{n}\right), v\right\rangle=\int_{\Omega} u_{n}^{\tau-1} v d x+\lambda_{n} \int_{\Omega} f\left(\cdot, u_{n}\right) v d x, \quad v \in X .
$$

We can also assume $\Phi_{\lambda}\left(u_{n}\right)<0$ (see the proof of Proposition 3.1), which means

$$
\left\|\nabla u_{n}\right\|_{p}^{p}+\frac{p}{q}\left\|\nabla u_{n}\right\|_{q}^{q}-\frac{p}{\tau}\left\|u_{n}\right\|_{\tau}^{\tau}-\lambda_{n} \int_{\Omega} p F\left(x, u_{n}(x)\right) d x<0 .
$$

Testing (3.8) with $v:=u_{n}$ gives

$$
\left\|\nabla u_{n}\right\|_{p}^{p}+\left\|\nabla u_{n}\right\|_{q}^{q}=\left\|u_{n}\right\|_{\tau}^{\tau}+\lambda_{n} \int_{\Omega} f\left(\cdot, u_{n}\right) u_{n} d x
$$

Since $q<p$ while $\lambda_{1} \leq \lambda_{n}$, from (3.9)-(3.10) it follows

$$
\int_{\Omega}\left[f\left(\cdot, u_{n}\right) u_{n}-p F\left(\cdot, u_{n}\right)\right] d x \leq \frac{1}{\lambda_{1}}\left(\frac{p}{\tau}-1\right)\left\|u_{n}\right\|_{\tau}^{\tau} \quad \forall n \in \mathbb{N} .
$$

Observe next that, thanks to $\left(\mathrm{h}_{1}\right)$ and $\left(\mathrm{h}_{3}\right)$, one has

$$
f(x, \xi) \xi-p F(x, \xi) \geq c_{8} \xi^{\beta}-c_{9} \quad \text { in } \quad \Omega \times \mathbb{R}_{+} .
$$

Consequently, (3.11) becomes

$$
c_{8}\left\|u_{n}\right\|_{\beta}^{\beta} \leq \frac{1}{\lambda_{1}}\left(\frac{p}{\tau}-1\right)\left\|u_{n}\right\|_{\tau}^{\tau}+c_{10} \leq c_{11}\left\|u_{n}\right\|_{\beta}^{\tau}+c_{10}, \quad n \in \mathbb{N},
$$

because $\tau<\beta$. This clearly forces

$$
\left\|u_{n}\right\|_{\beta} \leq c_{12} \quad \forall n \in \mathbb{N} .
$$

If $r \leq \beta$ then $\left\{u_{n}\right\}$ turns out also bounded in $L^{r}(\Omega)$. Using (3.10) besides $\left(\mathrm{h}_{1}\right)$ entails

$$
\begin{aligned}
\left\|u_{n}\right\|^{p} & \leq\left\|\nabla u_{n}\right\|_{p}^{p}+\left\|\nabla u_{n}\right\|_{q}^{q} \leq\left\|u_{n}\right\|_{\tau}^{\tau}+\lambda^{*} \int_{\Omega} f\left(\cdot, u_{n}\right) u_{n} d x \\
& \leq|\Omega|^{1-\tau / r}\left\|u_{n}\right\|_{r}^{\tau}+\lambda^{*} c_{0} \int_{\Omega}\left(u_{n}^{\theta}+u_{n}^{r}\right) d x \\
& \leq|\Omega|^{1-\tau / r}\left\|u_{n}\right\|_{r}^{\tau}+\lambda^{*} c_{0} \int_{\Omega}\left[\left(1+u_{n}^{r}\right)+u_{n}^{r}\right] d x,
\end{aligned}
$$

whence $\left\{u_{n}\right\} \subseteq X$ is bounded. Suppose now $\beta<r<p^{*}$. Two cases may occur.

1) $p<N$. Let $t \in(0,1)$ satisfy

$$
\frac{1}{r}=\frac{1-t}{\beta}+\frac{t}{p^{*}}
$$

The interpolation inequality [12, p. 905] yields $\left\|u_{n}\right\|_{r} \leq\left\|u_{n}\right\|_{\beta}^{1-t}\left\|u_{n}\right\|_{p^{*}}^{t}$. Via (3.12) we thus obtain

$$
\left\|u_{n}\right\|_{r}^{r} \leq c_{13}\left\|u_{n}\right\|_{p^{*}}^{t r}, \quad n \in \mathbb{N} .
$$


Reasoning exactly as before and exploiting (3.15) produces

$$
\left\|u_{n}\right\|^{p} \leq\left\|\nabla u_{n}\right\|_{p}^{p}+\left\|\nabla u_{n}\right\|_{q}^{q} \leq c_{14}\left(1+\left\|u_{n}\right\|_{p^{*}}^{t r}\right) \leq c_{15}\left(1+\left\|u_{n}\right\|^{t r}\right) .
$$

Finally, note that $t r<p$. Indeed, $(r-p) \frac{N}{p}<\beta$ due to $\left(\mathrm{h}_{3}\right)$, while

$$
t r<p \Longleftrightarrow \frac{r-\beta}{p^{*}-\beta}<\frac{p}{p^{*}} \Longleftrightarrow(r-p) \frac{N}{p}<\beta
$$

cf. (3.14). Now, the boundedness of $\left\{u_{n}\right\} \subseteq X$ directly stems from (3.16).

2) $p \geq N$, which implies $p^{*}=+\infty$. We will repeat the previous argument with $p^{*}$ replaced by any $\sigma>r$. Accordingly, if $t \in(0,1)$ fulfills $\frac{1}{r}=\frac{1-t}{\beta}+\frac{t}{\sigma}$ then $t r=\frac{\sigma(r-\beta)}{\sigma-\beta}$. Since, thanks to $\left(\mathrm{h}_{3}\right)$ again,

$$
\lim _{\sigma \rightarrow+\infty} \frac{\sigma(r-\beta)}{\sigma-\beta}=r-\beta<p,
$$

one arrives at $t r<p$ for $\sigma$ large enough. This entails $\left\{u_{n}\right\} \subseteq X$ bounded once more.

Hence, in either case, we may assume

$$
u_{n} \rightarrow u^{*} \text { in } X \text { and } u_{n} \rightarrow u^{*} \text { in } L^{r}(\Omega),
$$

where a subsequence is considered when necessary. Testing (3.8) with $v:=u_{n}-u^{*}$ thus yields, as $n \rightarrow+\infty$,

$$
\lim _{n \rightarrow+\infty}\left\langle A_{p}\left(u_{n}\right)+A_{q}\left(u_{n}\right), u_{n}-u^{*}\right\rangle=0
$$

whence, by monotonicity of $A_{q}$,

$$
\limsup _{n \rightarrow+\infty}\left[\left\langle A_{p}\left(u_{n}\right), u_{n}-u^{*}\right\rangle+\left\langle A_{q}(u), u_{n}-u^{*}\right\rangle\right] \leq 0 .
$$

On account of (3.17) it follows

$$
\limsup _{n \rightarrow+\infty}\left\langle A_{p}\left(u_{n}\right), u_{n}-u^{*}\right\rangle \leq 0 .
$$

Recalling that $A_{p}$ enjoys the $(\mathrm{S})_{+}$-property, we infer $u_{n} \rightarrow u^{*}$ in $X$, besides $0 \leq u_{n} \leq u^{*}$ for all $n \in \mathbb{N}$. Finally, let $n \rightarrow+\infty$ in (3.8) to get

$$
\left\langle A_{p}\left(u^{*}\right)+A_{q}\left(u^{*}\right), v\right\rangle=\int_{\Omega}\left(u^{*}\right)^{\tau-1} v d x+\lambda^{*} \int_{\Omega} f\left(\cdot, u^{*}\right) v d x \quad \forall v \in X,
$$

i.e., $u^{*} \in S_{\lambda^{*}}$ and, a fortiori, $\lambda^{*} \in \mathcal{L}$.

Some meaningful (bifurcation) properties of the set $S_{\lambda}$ will now be established.

Proposition 3.7. Suppose $\left(\mathrm{h}_{1}\right)-\left(\mathrm{h}_{4}\right)$ hold true. Then, for every $\lambda \in\left(0, \lambda^{*}\right)$, problem $\left(\mathrm{P}_{\lambda}\right)$ admits two solutions $u_{0}, \hat{u} \in \operatorname{int}\left(C_{+}\right)$such that $u_{0} \leq \hat{u}$. Moreover, $u_{0}$ is a local minimizer of the associated energy functional $\Phi_{\lambda}$.

Proof. Fix $\lambda \in\left(0, \lambda^{*}\right)$ and choose $\eta \in\left(\lambda, \lambda^{*}\right)$. By Proposition 3.2, there exists $u_{\eta} \in S_{\eta}$ while Proposition 3.4 provides $u_{0} \in S_{\lambda}$ satisfying

$$
u_{0} \in \operatorname{int}_{C_{0}^{1}(\bar{\Omega})}\left(\left[0, u_{\eta}\right]\right) .
$$

The same reasoning adopted in the proof of Proposition 3.2 ensures here that $u_{0}$ is a global minimum point to the functional

$$
\Phi_{\lambda, \eta}(u):=\frac{1}{p}\|\nabla u\|_{p}^{p}+\frac{1}{q}\|\nabla u\|_{q}^{q}-\int_{\Omega} F_{\lambda, \eta}(x, u(x)) d x, \quad u \in X,
$$

where $F_{\lambda, \eta}(x, \xi):=\int_{0}^{\xi} f_{\lambda, \eta}(x, t) d t$, with

$$
f_{\lambda, \eta}(x, \xi):= \begin{cases}\left(\xi^{+}\right)^{\tau-1}+\lambda f\left(x, \xi^{+}\right) & \text {if } \xi \leq u_{\eta}(x) \\ u_{\eta}(x)^{\tau-1}+\lambda f\left(x, u_{\eta}(x)\right) & \text { otherwise }\end{cases}
$$


By (3.18), $u_{0}$ turns out a local $C_{0}^{1}(\bar{\Omega})$-minimizer of $\Phi_{\lambda}$, because $\Phi_{\lambda}\left\lfloor_{\left[0, u_{\eta}\right]}=\Phi_{\lambda, \eta} L_{\left[0, u_{\eta}\right]}\right.$. Via Proposition 2.1 we then see that this remains valid with $C_{0}^{1}(\bar{\Omega})$ replaced by $X$. Set

$$
f_{0}(x, \xi):=\left\{\begin{array}{ll}
u_{0}(x)^{\tau-1}+\lambda f\left(x, u_{0}(x)\right) & \text { if } \xi \leq u_{0}(x), \\
\xi^{\tau-1}+\lambda f(x, \xi) & \text { otherwise, }
\end{array} \quad F_{0}(x, \xi):=\int_{0}^{\xi} f_{0}(x, t) d t,\right.
$$

$(x, \xi) \in \Omega \times \mathbb{R}$, as well as

$$
\Phi_{0}(u):=\frac{1}{p}\|\nabla u\|_{p}^{p}+\frac{1}{q}\|\nabla u\|_{q}^{q}-\int_{\Omega} F_{0}(x, u(x)) d x \quad \forall u \in X .
$$

From (3.19) and the nonlinear regularity theory it follows $u_{0} \in K\left(\Phi_{0}\right) \subseteq\left[u_{0}\right) \cap \operatorname{int}\left(C_{+}\right)$. We may thus assume

$$
K\left(\Phi_{0}\right) \cap\left[u_{0}, u_{\eta}\right]=\left\{u_{0}\right\},
$$

or else a second solution of $\left(\mathrm{P}_{\lambda}\right)$ bigger than $u_{0}$ would exist. Bearing in mind the proof of Proposition 3.6 and making small changes to accommodate the truncation at $u_{0}(x)$ shows that $\Phi_{0}$ satisfies condition (C). Let us next truncate $f_{0}(x, \cdot)$ at $u_{\eta}(x)$ to construct a new Carathéodory function $\tilde{f}$, with primitive $\tilde{F}$ and associated functional $\tilde{\Phi}$, defined like in (3.20) but replacing $F_{0}$ by $\tilde{F}$. Evidently,

$$
K(\tilde{\Phi})=K\left(\Phi_{0}\right) \cap\left[u_{0}, u_{\eta}\right],
$$

whence $K(\tilde{\Phi})=\left\{u_{0}\right\}$ because of (3.21). Since $\tilde{\Phi}$ is coercive and weakly sequentially lower semicontinuous, it possesses a global minimum point that must coincide with $u_{0}$. An easy verification gives $\Phi_{0} L_{\left[0, u_{\eta}\right]}=\tilde{\Phi} L_{\left[0, u_{\eta}\right]}$. So, thanks to (3.18), $u_{0}$ turns out a local $C_{0}^{1}(\bar{\Omega})$-minimizer of $\Phi_{0}$. This still holds when $X$ replaces $C_{0}^{1}(\bar{\Omega})$; cf. Proposition 2.1. We may suppose $K\left(\Phi_{0}\right)$ finite, otherwise infinitely many solutions of $\left(\mathrm{P}_{\lambda}\right)$ bigger than $u_{0}$ do exist. Adapting the argument exploited in [1, Proposition 29] provides $\rho \in(0,1)$ such that

$$
\Phi_{0}\left(u_{0}\right)<m_{0}:=\inf \left\{\Phi_{0}(u):\left\|u-u_{0}\right\|=\rho\right\} .
$$

Finally, if $u \in \operatorname{int}\left(C_{+}\right)$then simple calculations based on $\left(\mathrm{h}_{2}\right)$ entail $\Phi_{0}(t u) \rightarrow-\infty$ as $t \rightarrow+\infty$. Therefore, the mountain pass theorem can be applied, and there is $\hat{u} \in X$ fulfilling

$$
\hat{u} \in K\left(\Phi_{0}\right), \quad \Phi_{0}(\hat{u}) \geq m_{0} .
$$

Via (3.22)-(3.23) one has $u_{0} \neq \hat{u}$ while the inclusion $K\left(\Phi_{0}\right) \subseteq\left[u_{0}\right) \cap \operatorname{int}\left(C_{+}\right)$forces $u_{0} \leq \hat{u}$, which ends the proof.

Proposition 3.8. Under $\left(\mathrm{h}_{1}\right)-\left(\mathrm{h}_{4}\right)$, the solution set $S_{\lambda}$ admits a smallest element $\bar{u}_{\lambda}$ for every $\lambda \in \mathcal{L}$.

Proof. A standard procedure ensures that $S_{\lambda}$ turns out downward directed; see, e.g., [10, Section 4]. Lemma 3.10 at p. 178 of [17] yields

$$
\text { ess } \inf S_{\lambda}=\inf \left\{u_{n}: n \in \mathbb{N}\right\}
$$

for some decreasing sequence $\left\{u_{n}\right\} \subseteq S_{\lambda}$. Consequently, $0 \leq u_{n} \leq u_{1}$ and

$$
\left\langle A_{p}\left(u_{n}\right)+A_{q}\left(u_{n}\right), v\right\rangle=\int_{\Omega}\left[u_{n}^{\tau-1}+\lambda f\left(\cdot, u_{n}\right)\right] v d x \quad \forall v \in X .
$$

Due to $\left(\mathrm{h}_{1}\right)$, testing $(3.25)$ with $v:=u_{n}$ we thus obtain

$$
\begin{aligned}
\left\|u_{n}\right\|^{p} & \leq\left\|\nabla u_{n}\right\|_{p}^{p}+\left\|\nabla u_{n}\right\|_{q}^{q}=\int_{\Omega}\left[u_{n}^{\tau}+\lambda f\left(\cdot, u_{n}\right) u_{n}\right] d x \\
& \leq \int_{\Omega}\left[u_{n}^{\tau}+\lambda c_{0}\left(u_{n}^{\theta}+u_{n}^{r}\right)\right] d x \leq \int_{\Omega}\left[u_{1}^{\tau}+\lambda c_{0}\left(u_{1}^{\theta}+u_{1}^{r}\right)\right] d x, \quad n \in \mathbb{N},
\end{aligned}
$$

namely $\left\{u_{n}\right\} \subseteq X$ is bounded. Like before (cf. the proof of Proposition 3.6), this gives $u_{n} \rightarrow \bar{u}_{\lambda}$ in $X$, where a subsequence is considered if necessary. So, from (3.25) it easily follows

$$
\left\langle A_{p}\left(\bar{u}_{\lambda}\right)+A_{q}\left(\bar{u}_{\lambda}\right), v\right\rangle=\int_{\Omega}\left[\bar{u}_{\lambda}^{\tau-1}+\lambda f\left(\cdot, \bar{u}_{\lambda}\right)\right] v d x \quad \forall v \in X .
$$


Showing that $\bar{u}_{\lambda} \neq 0$ will entail $\bar{u}_{\lambda} \in S_{\lambda}$, whence the conclusion by (3.24). To the aim, consider the problem

$$
-\Delta_{p} u-\Delta_{q} u=u^{\tau-1} \text { in } \Omega, \quad u>0 \text { in } \Omega, \quad u=0 \text { on } \partial \Omega .
$$

Its energy functional

$$
\Phi_{0}(u):=\frac{1}{p}\|\nabla u\|_{p}^{p}+\frac{1}{q}\|\nabla u\|_{q}^{q}-\frac{1}{\tau}\left\|u^{+}\right\|_{\tau}^{\tau}, \quad u \in X,
$$

turns out coercive and weakly sequentially lower semicontinuous. Hence, there exists $\tilde{u} \in X$ satisfying $\Phi_{0}(\tilde{u})=\inf _{X} \Phi_{0}$. One has $u_{0} \neq 0$, because $\Phi_{0}(\tilde{u})<0=\Phi_{0}(0)$ (the argument is like in the proof of Proposition 3.5). Further, $\Phi_{0}^{\prime}(\tilde{u})=0$, i.e.,

$$
\left\langle A_{p}(\tilde{u})+A_{q}(\tilde{u}), v\right\rangle=\int_{\Omega}\left(\tilde{u}^{+}\right)^{\tau-1} v d x \quad \forall v \in X .
$$

Choosing $v:=-\tilde{u}^{-}$we see that $u$ is a positive solution to (3.26). Actually, $\tilde{u} \in \operatorname{int}\left(C_{+}\right)$and, through a standard procedure [15, Lemma 3.1], $\tilde{u}$ turns out unique.

Claim: $\tilde{u} \leq u$ for all $u \in S_{\lambda}$.

Indeed, fixed any $u \in S_{\lambda}$, define

$$
\Psi(w):=\frac{1}{p}\|\nabla u\|_{p}^{p}+\frac{1}{q}\|\nabla u\|_{q}^{q}-\int_{\Omega} d x \int_{0}^{w(x)} g(x, t) d t, \quad w \in X,
$$

where

$$
g(x, t):=\left\{\begin{array}{ll}
\left(t^{+}\right)^{\tau-1} & \text { if } t \leq u(x), \\
u(x)^{\tau-1} & \text { otherwise }
\end{array} \quad \forall(x, t) \in \Omega \times \mathbb{R} .\right.
$$

The following assertions can be easily verified.

- $\Psi\left(u^{*}\right)=\inf _{X} \Psi$, with appropriate $u^{*} \in X$.

- $\Psi\left(u^{*}\right)<0=\Psi(0)$, whence $u^{*} \neq 0$.

- $u^{*} \in K(\Psi) \subseteq[0, u] \cap C_{+}$.

Therefore, $u^{*}$ is a positive solution of (3.26). By uniqueness, this implies $u^{*}=\tilde{u}$. Thus, a fortiori, $\tilde{u} \leq u$.

The claim brings $\tilde{u} \leq u_{n}, n \in \mathbb{N}$, which in turn provides $0<\tilde{u} \leq \bar{u}_{\lambda}$, as desired.

Let us finally come to some meaningful properties of the map

$$
k: \lambda \in \mathcal{L} \mapsto \bar{u}_{\lambda} \in C_{0}^{1}(\bar{\Omega}) .
$$

Proposition 3.9. Suppose $\left(\mathrm{h}_{1}\right)-\left(\mathrm{h}_{4}\right)$ hold true. Then the function $k$ is both

$\left(i_{1}\right)$ strictly increasing, namely $\bar{u}_{\lambda_{2}}-\bar{u}_{\lambda_{1}} \in \operatorname{int}\left(C_{+}\right)$if $\lambda_{1}<\lambda_{2}$, and

$\left(\mathrm{i}_{2}\right)$ left-continuous.

Proof. Pick $\lambda_{1}, \lambda_{2} \in \mathcal{L}$ such that $\lambda_{1}<\lambda_{2}$. Since $\bar{u}_{\lambda_{2}} \in S_{\lambda_{2}}$, Proposition 3.4 yields $u_{\lambda_{1}} \in S_{\lambda_{1}}$ fulfilling $\bar{u}_{\lambda_{2}}-u_{\lambda_{1}} \in \operatorname{int}\left(C_{+}\right)$, while Proposition 3.8 entails $\bar{u}_{\lambda_{1}} \leq u_{\lambda_{1}}$. Hence, $\bar{u}_{\lambda_{2}}-\bar{u}_{\lambda_{1}} \in \operatorname{int}\left(C_{+}\right)$. This shows $\left(\mathrm{i}_{1}\right)$.

If $\lambda_{n} \rightarrow \lambda^{-}$in $\mathcal{L}$ then, by (i $\left.i_{1}\right)$, the sequence $\left\{\bar{u}_{\lambda_{n}}\right\}$ turns out increasing. Its boundedness in $X$ immediately stems from $\left(\mathrm{h}_{1}\right)$; see the previous proof. Now, repeat the argument below (3.17) to arrive at

$$
\bar{u}_{\lambda_{n}} \rightarrow \tilde{u}_{\lambda} \text { in } X \text {, }
$$

whence $\tilde{u}_{\lambda} \in S_{\lambda} \subseteq \operatorname{int}\left(C_{+}\right)$. We finally claim that $\tilde{u}_{\lambda}=\bar{u}_{\lambda}$. Assume on the contrary

$$
\bar{u}_{\lambda}\left(x_{0}\right)<\tilde{u}_{\lambda}\left(x_{0}\right) \text { for some } x_{0} \in \Omega \text {. }
$$

Lieberman's nonlinear regularity theory gives $\left\{\bar{u}_{n}\right\} \subseteq C_{0}^{1, \alpha}(\bar{\Omega})$ as well as

$$
\left\|\bar{u}_{\lambda_{n}}\right\|_{C_{0}^{1, \alpha}(\bar{\Omega})} \leq c_{16} \quad \forall n \in \mathbb{N} \text {. }
$$


Since the embedding $C_{0}^{1, \alpha}(\bar{\Omega}) \hookrightarrow C_{0}^{1}(\bar{\Omega})$ is compact, (3.27) becomes

$$
\bar{u}_{\lambda_{n}} \rightarrow \tilde{u}_{\lambda} \text { in } C_{0}^{1}(\bar{\Omega}) \text {. }
$$

Because of (3.28), this implies $\bar{u}_{\lambda}\left(x_{0}\right)<\bar{u}_{\lambda_{n}}\left(x_{0}\right)$ for any $n$ large enough, against (i $\left.i_{1}\right)$. Consequently, $\tilde{u}_{\lambda}=\bar{u}_{\lambda}$, and (i $\left.\mathrm{i}_{2}\right)$ follows from (3.27).

Gathering Propositions 3.1-3.9 together we obtain the following

Theorem 3.10. Let $\left(\mathrm{h}_{1}\right)-\left(\mathrm{h}_{4}\right)$ be satisfied. Then, there exists $\lambda^{*}>0$ such that problem $\left(\mathrm{P}_{\lambda}\right)$ admits

( $\left.\mathrm{j}_{1}\right)$ at least two solutions $u_{0}, \hat{u} \in \operatorname{int}\left(C_{+}\right)$, with $u_{0} \leq \hat{u}$, for every $\lambda \in\left(0, \lambda^{*}\right)$,

$\left(\mathrm{j}_{2}\right)$ at least one solution $u^{*} \in \operatorname{int}\left(C_{+}\right)$when $\lambda=\lambda^{*}$,

$\left(\mathrm{j}_{3}\right)$ no positive solutions for all $\lambda>\lambda^{*}$,

(j4) a smallest positive solution $\bar{u}_{\lambda} \in \operatorname{int}\left(C_{+}\right)$provided $\lambda \in\left(0, \lambda^{*}\right]$.

Moreover, the map $\lambda \in\left(0, \lambda^{*}\right] \mapsto \bar{u}_{\lambda} \in C_{0}^{1}(\bar{\Omega})$ is strictly increasing and left-continuous.

ACKnowledgment. This work is performed within the 2016-2018 Research Plan - Intervention

Line 2: 'Variational Methods and Differential Equations', and partially supported by GNAMPA of INDAM.

\section{REFERENCES}

[1] S. Aizicovici, N.S. Papageorgiou and V. Staicu, Degree theory for operators of monotone type and nonlinear elliptic equations with inequality constraints, Mem. Amer. Math. Soc. 196 (2008). 9

[2] A. Ambrosetti, H. Brezis, and G. Cerami, Combined effects of concave and convex nonlinearities in some elliptic problems, J. Funct. Anal. 122 (1994), 519-543 1

[3] D. Arcoya and D. Ruiz, The Ambrosetti-Prodi problem for the p-Laplace operator, Comm. Partial Differential Equations 31 (2006), 849-865. 3

[4] G. Barletta, R. Livrea, and N.S. Papageorgiou, Bifurcation phenomena for the positive solutions of semilinear elliptic problems with mixed boundary conditions, J. Nonlinear Convex Anal. 17 (2016), 1497-1516. 2

[5] V. Bobkov and M. Tanaka, On positive solutions for $(p, q)$-Laplace equations with two parameters, Calc. Var. Partial Differential Equations 54 (2015), 3277-3301. 7

[6] V. Bobkov and M. Tanaka, Remarks on minimizers for $(p, q)$-Laplace equations with two parameters, Commun. Pure Appl. Anal. 17 (2018), 1219-1253. 3

[7] L. Cherfils and Y. Ilyasov, On the stationary solutions of generalized reaction diffusion equations with $(p, q)$ Laplacian, Comm. Pure Appl. Anal. 4 (2005), 9-22. 1

[8] G. H. Derrick, Comments on nonlinear wave equations as models for elementary particles, J. Math. Phys. 5 (1964), 1252-1254. 1

[9] P.C. Fife, Mathematical Aspects of Reacting and Diffusing Systems, Lect. Notes in Biomath. 28, Springer, Berlin, 1979. 1

[10] M. Filippakis and N.S. Papageorgiou, Multiple constant sign and nodal solutions for nonlinear elliptic equations with the p-Laplacian, J. Differential Equations 245 (2008), 1883-1922. 9

[11] J. Garcia Azorero, J. Manfredi, and I. Peral Alonso, Sobolev versus Hölder local minimizers and global multiplicity for some quasilinear elliptic equations, Comm. Contemp. Math. 2 (2000), 385-404. 1

[12] L. Gasiński and N.S. Papageorgiou, Nonlinear Analysis, Chapman \& Hall, CRC, Boca Raton, Fl, 2006. 3,7

[13] L. Gasiński and N.S. Papageorgiou, Multiple solutions for nonlinear coercive problems with a nonhomogeneous differential operator and a nonsmooth potential, Set-Valued Anal. 20 (2012), 417-443. 3

[14] L. Gasiński and N.S. Papageorgiou, Bifurcation-type results for nonlinear parametric elliptic equations, Proc. Royal Soc. Edinburgh Sect. A 142 (2012), 595-623. 2

[15] U. Guarnotta, S.A. Marano, and N.S. Papageorgiou, Multiple nodal solutions to a Robin problem with sign-changing potential and locally defined reaction, Atti Accad. Naz. Lincei Rend. Lincei Mat. Appl., in press. 10

[16] Z. Guo and Z. Zhang, $W^{1, p}$ versus $C^{1}$ local minimizers and multiplicity results for quasilinear elliptic equations, J. Math. Anal. Appl. 286 (2003), 32-50. 1

[17] S. Hu and N.S. Papageorgiou, Handbook of Multivalued Analysis. Volume I: Theory, Kluwer Academic Publishers, Dordrecht, The Netherlands, 1997. 9

[18] G. Lieberman, The natural generalization of the natural conditions of Ladyshenskaya and Ural'tseva for elliptic equations, Comm. Partial Differential Equations 16 (1991), 311-361. 4

[19] S.A. Marano and S. Mosconi, Some recent results on the Dirichlet problem for $(p, q)$-Laplace equations, Discrete Contin. Dyn. Syst. Ser. S 11 (2018), 279-291. 1

[20] S.A. Marano, S. Mosconi, and N.S. Papageorgiou, Multiple solutions to $(p, q)$-Laplacian problems with resonant concave nonlinearity, Adv. Nonlinear Stud. 16 (2016), 51-65. 2 
[21] S.A. Marano, S. Mosconi, and N.S. Papageorgiou, On a $(p, q)$-Laplacian problem with concave and asymmetric perturbation, Atti Accad. Naz. Lincei Rend. Lincei Mat. Appl. 29 (2018), 109-125. 2

[22] S.A. Marano and N.S. Papageorgiou, Positive solutions to a Dirichlet problem with p-Laplacian and concave-convex nonlinearity depending on a parameter, Comm. Pure Appl. Anal. 12 (2013), 815-829. 1, 6

[23] N.S. Papageorgiou and V.D. Radulescu, Bifurcation of positive solutions for nonlinear nonhomogeneous Robin and Neumann problems with competing nonlinearities, Discrete Contin. Dyn. Syst.-A 35 (2016), 5008-5036. 2

[24] N.S. Papageorgiou, V.D. Radulescu, and D. Repovs, Robin problems with indefinite linear part and competition phenomena, Commun. Pure Appl. Anal. 16 (2017), 1293-1314. 1

[25] P. Pucci and J. Serrin, The Maximum Principle, Birkhäuser, Basel, 2007. 4

[26] H. Wilhelmson, Explosive instabilities of reaction-diffusion equations, Phys. Rev. A 36 (1987), 965-966. 1

[27] V.V. Zhikov, Averaging of functionals of the calculus of variations and elasticity theory, Izv. Akad. Nauk SSSR Ser. Mat. 50 (1986), 675-710 ; English translation in Math. USSR-Izv. 29 (1987), 33-66. 1

Salvatore A. Marano, Dipartimento di Matematica e Informatica, Università degli Studi di Catania Viale

A. Doria 6, 95125 Catania, Italy

E-mail address: marano@dmi.unict.it

Greta Marino, Dipartimento di Matematica e Informatica, Università degli Studi di Catania, Viale A. Doria 6, 95125 Catania, Italy

E-mail address: greta.marino@dmi.unict.it

Nikolaos S. Papageorgiou, Department of Mathematics, National Technical University, Zografou CamPus, 15780 Athens, Greece

E-mail address: npapg@math.ntua.gr 